\title{
Comportamento alimentar e promoção da saúde
}

\author{
Patrícia Radaelli ${ }^{1}$ \\ Elisabetta Recine ${ }^{2}$
}

\begin{abstract}
RESUMO - O presente artigo consiste em revisão de literatura sobre temas relacionados com o comportamento alimentar e a promoção da saúde. São discutidas experiências de diferentes países em promover saúde por meio da alimentação equilibrada, os principais benefícios e as barreiras em seguir alimentação saudável, as influências no comportamento alimentar e as tendências atuais de consumo de alimentos da população brasileira.
\end{abstract}

Palavras-chave: comportamento alimentar, promoção da saúde, alimentação saudável, consumo de alimentos.

\section{Eating behavior and health promotion}

\begin{abstract}
This article reviews eating behavior and health promotion subjects. Different countries experiences to promote health by a healthy eating, benefits and barriers in trying to eat healthier, influences on food choices and contemporary food consumption tendencies of Brazilian population are discussed.
\end{abstract}

Key words: eating behavior, health promotion, healthy eating, food consumption.

O aumento da morbidade e da mortalidade por doenças crônico-degenerativas relacionadas à alimentação, a exemplo da obesidade e da hipertensão arterial, é conhecido como transição epidemiológica alimentar e nutricional (OLIVEIRA et al., 1996; MONDINI \& MONTEIRO, 2000). No Brasil, isto pôde ser evidenciado

\footnotetext{
${ }^{1}$ Mestre em Nutrição Humana pela UnB e professora de Nutrição do UniCEUB. E-mail: patricia_radaelli@ig.com.br

${ }^{2}$ Doutora em Saúde Pública pela USP e professora de Nutrição da UnB. E-mail: erecine@adinet.com.uy

O presente artigo foi baseado em dissertação de Mestrado da primeira autora, aprovada em 2003, sob orientação da segunda autora.
} 
mediante análise comparativa entre dois inquéritos nacionais ocorridos em 1974 e 1989, o que demonstrou crescimento do número de adultos obesos correspondente a 75\% de homens e 60\% de mulheres (OLIVEIRA et al., 1996; DOYLE \& FELDMAN, 1997; TADDEI, 1998; MONTEIRO et al., 2000; BARRETO \& CYRILLO, 2001).

Os dados de obesidade na população adulta brasileira são provenientes da Pesquisa Nacional sobre Saúde e Nutrição (PNSN), realizada em 1989. Estimou-se que cerca de 27 milhões de indivíduos maiores de 18 anos de idade, ou seja, 32\% da população adulta apresentam algum grau de excesso de peso, isto é, um Índice de Massa Corporal (IMC) maior ou igual a $25 \mathrm{~kg} / \mathrm{m}^{2}$ (OLIVEIRA et al., 1996).

Desagregados por sexo, os dados da PNSN indicam que 27\% dos homens e $38 \%$ das mulheres apresentam sobrepeso ou obesidade. Com relação à obesidade em adultos (IMC e" $30 \mathrm{~kg} / \mathrm{m}^{2}$ ), observou-se a prevalência de $8 \%$ na população total, sendo que $70 \%$ ocorre em mulheres. Considerando-se o excesso de peso relacionado às faixas de renda, de forma global, as prevalências tendem a ser mais elevadas em populações de renda mais alta. No entanto, particularmente na população feminina, a freqüência de excesso de peso é expressiva também entre faixas de menor renda. Entre mulheres pertencentes a faixas de menor poder aquisitivo (d" 5 salários-mínimos per capita), cerca de $30 \%$ apresentam excesso de peso (MS, 2002a).

A obesidade tornou-se preocupação de saúde pública tanto quanto a desnutrição, e tal conjuntura confere ao País perfil epidemiológico polarizado e com extrema desigualdade, cujas causas e conseqüências devem ser cuidadosamente analisadas para orientar as políticas de saúde e nutrição. Os dados diretamente relacionados com o estado nutricional expressam realidade social de extrema gravidade, cuja perspectiva futura é preocupante (GALEAZZI et al., 1997). Em todas as regiões do País, parcelas expressivas da população adulta apresentam excesso de peso, mas as menores prevalências são encontradas na Região Nordeste e as maiores, na Região Sudeste (MS, 2002a).

Encontrar estratégia para melhorar a qualidade da dieta é responsabilidade de políticos e de promotores de saúde. Divulgar informações consiste em uma das estratégias, mas não atinge os objetivos de maneira isolada, uma vez que os hábitos alimentares dependem de fatores não somente relativos ao indivíduo, mas também sociais, culturais, econômicos e ambientais (VAANDRAGER \& KOELEN, 1997). Esses fatores estão interligados, e a escolha de alimentos consiste em processo complexo, o que justifica, em parte, a ineficácia de se promover alimentação saudável apenas com o elemento cognitivo (ADA, 1996).

Indubitavelmente, a identificação do comportamento alimentar de indivíduos é essencial para se priorizar programas de promoção de saúde viáveis e efica- 
zes. Se, no Brasil, os dados sobre consumo de alimentos são escassos, as informações sobre o comportamento alimentar são praticamente inexistentes, o que torna cada vez mais indispensável a adoção de abordagem multidisciplinar e comparativa em que os fatores associados às atitudes com relação aos alimentos sejam analisados de maneira que permitam a elucidação dos mecanismos responsáveis pela formação e, mais ainda, pela transformação de hábitos alimentares e suas conseqüências em diferentes contextos socioeconômicos encontrados no País.

\section{Guias alimentares: experiências na promoção de orientações nutricionais}

A inter-relação entre hábitos alimentares, manutenção da saúde e desenvolvimento de doenças crônicas tornou-se evidente nos últimos trinta anos, e estudos prospectivos dão grande contribuição nesse aspecto (WHO/FAO, 2003). O reconhecimento de que características da dieta podem exercer influência decisiva sobre o estado de saúde dos indivíduos determinou que a Organização Mundial de Saúde estabelecesse guias alimentares que definissem limites seguros para consumo de gorduras, colesterol, açúcar, entre outras substâncias (WHO, 1990).

Muitos países preocupam-se em desenvolver orientações nutricionais para encorajar a população a adotar hábitos alimentares saudáveis. Essas orientações costumam enfatizar os princípios de variedade, moderação e equilíbrio de alimentos, além de incentivar o consumo de fibras e de alimentos reguladores e recomendar redução na ingestão de gorduras (CONTENTO et al., 2002). Entretanto, pesquisas revelam sucesso limitado na adoção desses princípios, confirmado pelo aumento da prevalência de doenças crônico-degenerativas associadas à alimentação.

A Finlândia possui experiência positiva de promoção de hábitos saudáveis para a prevenção, em longo prazo, de doenças cardiovasculares (Puska et al., 1998). Durante o período de 1972 a 1997, foram desenvolvidas ações para alterar positivamente os hábitos alimentares de finlandeses, o que deu suporte à constatação de que as taxas de doenças cardiovasculares (DCVs) na população podem ser substancialmente influenciadas pela alimentação (PUSKA, 2000), uma vez que a prevalência desses tipos de patologias apresentou uma redução de 60\% (WHO, 2002). Além disso, a mortalidade por diferentes tipos de cânceres reduziu-se em 44\% (MS, 2002b).

Embora os Estados Unidos sejam atuantes na promoção de bons hábitos de saúde, a epidemia de obesidade nesse país é ocasionada, principalmente, por influências do meio social que promovem ingestão excessiva de alimentos e 
desencorajam a atividade física. Tendências contemporâneas de consumo de alimentos que incluem tipos de alimentos ingeridos e locais de alimentação são influenciadas por fatores sociais, culturais e econômicos (FRENCH et al., 2001). A saúde pública deve intervir com estratégias que promovam ambientes saudáveis quanto à alimentação e à prática de atividade física.

Assim, parece existir grande diferença entre o que é recomendado por profissionais da área e o que tem sido alcançado em termos de comportamento alimentar pela população (KOIKKALAINEN et al., 1996; GIBNEY et al., 1997; ANDING et al., 2001). Em relação à realidade norte-americana, por exemplo, há imensa disponibilidade de informações sobre alimentação e nutrição complexas, dispersas ou contraditórias, o que pode contribuir para a imobilização da população (IFIC, 1998). Variáveis relacionadas a atitudes, como a compreensão dos benefícios da alimentação saudável, incluindo a visão crítica sobre a dieta, e as dificuldades ou as barreiras em obtê-la também devem ser consideradas ao analisar a diferença entre o que as pessoas consomem e o que são aconselhadas a consumir (LENNERNÄS et al., 1997; ZUNFT et al., 1997; LAPPALAINEN et al., 1997).

Em tempos passados, as orientações nutricionais pareciam não atribuir a importância cabível a aspectos, como compreensão, atitudes e crenças relacionadas a alimentos por parte dos indivíduos (GIBNEY et al., 1997). Apenas na década de 90, durante a Conferência Internacional em Nutrição, explicitou-se a necessidade de compreender e de assimilar aspectos cognitivos e psicossociais referentes à alimentação e à saúde para a elaboração e implementação efetiva de guias alimentares e promoção de educação nutricional (FAO/WHO, 1992).

Atualmente, programas sobre alimentação saudável abordam temas práticos, como alimentação fora de casa ou em eventos sociais, maneiras simples de se tornar mais ativo, opções de boas escolhas de alimentos, considerando novas tendências de consumo, a exemplo de alimentos reduzidos em calorias (WHO/FAO, 2003). Reconhecidamente, a educação nutricional tende a ser mais efetiva quando centralizada no comportamento alimentar (CONTENTO et al., 2002), mas, na prática, isso ainda é observado com pouca freqüência.

O Ministério da Saúde aprovou, em 1999, a Política Nacional de Alimentação e Nutrição (PNAN) que apresenta, como uma de suas diretrizes, a Promoção de Práticas Alimentares e Estilos de Vida Saudáveis, com o objetivo de socializar o conhecimento sobre os alimentos e o processo de alimentação, bem como a prevenção dos problemas nutricionais. Inserido nesta diretriz, encontra-se o Guia Alimentar da População Brasileira, ainda não lançado, que será a base de ação dos profissionais de saúde na orientação alimentar da população com a perspectiva de promoção de saúde (CFN, 2003). 


\section{Benefícios e barreiras em seguir uma alimentação saudável}

O aumento da prevalência de doenças crônico-degenerativas relacionadas à alimentação, em especial a obesidade, impulsionou países europeus, Estados Unidos, Canadá, Austrália, entre outros, a desenvolver políticas com o objetivo de reverter esse quadro. A divulgação dos benefícios da dieta equilibrada foi uma das consequiências de tais políticas, e o acesso à informação facilitado pela mídia proporcionou maior alcance das informações, que se deu também em outros países. Outro efeito importante consistiu no reconhecimento da população sobre a relação positiva entre a dieta equilibrada e a prevenção de doenças, além da promoção da saúde (KRISTAL et al., 1995; LAPPALAINEN et al., 1997; USDA, 1998).

Contudo, os guias alimentares existentes são, geralmente, elaborados com pouca ou nenhuma consideração quanto às atitudes e percepções da população. A abordagem restrita pode ser uma das razões do sucesso limitado na modificação de hábitos alimentares (KEARNEY \& MCELHONE, 1999).

Além disso, o reconhecimento dos benefícios da alimentação equilibrada não é suficiente para a adesão a esse tipo de dieta, e são inúmeros os motivos pelos quais o indivíduo pode não seguir a orientação alimentar, tais como: conhecimento ou informação insuficiente, falta de interesse em modificar hábitos, influência de outras pessoas, presença de fatores identificados como obstáculos para seguir a dieta equilibrada, como escassez de dinheiro, de tempo ou de disponibilidade de alimentos saudáveis ou por esses não serem considerados saborosos e atrativos (MORREALE \& SCHWARTZ, 1995; DE CASTRO, 1997; MILLIGAN et al., 1997; THOMPSON et al., 1999). Smith e Owen (1992) citam, ainda, fatores emocionais, preocupação, ansiedade, cansaço e o estar sozinho, como limitantes da alimentação saudável. A falta de consenso nas informações disponíveis sobre alimentação também é barreira reconhecida para a adesão à alimentação equilibrada (COTUGNA et al., 1992; USDA, 1998; CASOTTI et al., 1998). As freqüentes mensagens divulgadas na mídia também contribuíram para isso (GOLDBERG, 2000).

As dificuldades relativas a situações sociais denotam a importância desses fatores, tratando-se de alimentação (MENNEL et al., 1992). Indivíduos que modificaram a dieta relatam que relações sociais podem impedir a manutenção dos novos hábitos alimentares (HOLM, 1993; KOIKKALAINEN et al., 1996). O conhecimento dos benefícios e das barreiras considerados pela população para seguir uma dieta equilibrada pode aumentar a probabilidade de estratégias que viabilizem a adoção de hábitos alimentares saudáveis. 


\section{Influências no comportamento alimentar, na compra e na esco- lha de alimentos}

O universo das práticas alimentares influencia o comportamento por meio de diversos fatores, entre os quais se destacam: qualidade e sabor dos alimentos, tradição alimentar, custo e disponibilidade de alimentos, preferências da família e de amigos, estrutura familiar, conveniência no preparo, apresentação do produto, religião e práticas ou objetivos alimentares específicos, como vegetarianismo, alimentação saudável e emagrecimento (RANDALL \& SANJUR, 1981; SHEPHERD, 1989; STEPTOE et al., 1995; LENNERNÄS et al., 1997; ORNELLAS, 2000; WHO/ FAO, 2003). A publicidade e a dinâmica de vida dos indivíduos também exercem papel importante nas decisões de consumo alimentar (BARRETO \& CYRILLO, 2001). Características, como poder aquisitivo, nível de escolaridade, ocupação, sexo, idade, peso, também afetam de maneira importante a escolha e a compra de alimentos (Tabela 1) (ROZIN, 1998; ROZIN \& SCHULKIN, 1998; MENNELL et al., 1992; KELDER et al., 1995; JEFFERY \& FRENCH, 1996; GROGAN et al., 1997; SPEAR \& KULBOK, 2001).

Estudos qualitativos apontam as experiências ao longo da vida do indivíduo como determinantes das escolhas alimentares, reconhecido como complexo, multidimensional, dinâmico e situacional, que consiste na categorização dos alimentos, na priorização de conflitos de valores em situações específicas e nas estratégias para equilibrar esses conflitos, o que inclui objetivos pessoais, questões ambientais, socioculturais, psicológicas, emocionais, de saúde e contexto da alimentação.

O indivíduo classifica os alimentos em diferentes categorias como "bom", "ruim", "saudável”, "saboroso", "caro", entre outras. Em determinada ocasião, pode-se ter como valor dominante o sabor e o prazer e, em outra, a saúde, a questão financeira ou a praticidade. As estratégias para equilibrar esses conflitos são as resultantes das escolhas (CONNORS et al., 2001).

A descrição detalhada das influências na escolha e na compra de alimentos pode auxiliar na seleção de alternativas mais apropriadas de promoção da alimentação saudável, além de ampliar as condições nas quais as estratégias utilizadas poderão ser mais bem-sucedidas (Lennernäs et al., 1997; Freeland-Graves \& Nitzke, 2002).

\section{Tendências atuais de consumo de alimentos}

Alterações de hábitos alimentares ocorrem nas últimas décadas, em vários países e estão associadas a fatores demográficos, econômicos, sociais 
epidemiológicos e nutricionais (DREWNOWSKI \& POPKIN, 1997; DE OLIVEIRA \& THEBAUD-MONY, 1997; MONTEIRO et al., 2000). Os dados nacionais disponíveis e mais abrangentes sobre consumo de alimentos correspondem aos resultados das Pesquisas de Orçamento Familiar (POF), conduzidas pela Fundação Getúlio Vargas em 1961/1963 e pelo Instituto Brasileiro de Geografia e Estatística (IBGE) em 1987/1988 e, mais recentemente, em 1995/1996, e do Estudo Nacional sobre Despesa Familiar (ENDEF), conduzida pelo IBGE em 1974/1975 (MS et al., 1990; OLIVEIRA, 1997).

Tais documentos revelam como principais tendências o decréscimo no consumo de alimentos, como mandioca, feijões, milho e arroz (OLIVEIRA et al., 1996). Outros produtos, como a carne de porco e a manteiga, vêm sendo consumidos em quantidades menores. A carne bovina tem sido substituída por carne de frango com mais freqüência, e ocorreu aumento da ingestão de ovos, laticínios e óleos vegetais. Essas pesquisas indicam declínio na ingestão de carboidratos, aumento de consumo de lipídeos como fonte de calorias, aumento no consumo de proteína animal, em detrimento da proteína vegetal, e aumento no consumo de alimentos industrializados (OLIVEIRA, 1997).

De menor abrangência, o Estudo Multicêntrico de Consumo Familiar de Alimentos foi realizado em Goiânia, Campinas, Ouro Preto e Curitiba e no Rio de Janeiro, entre os anos de 1996 e 1997, com o objetivo de identificar o perfil alimentar e nutricional dessas populações. Os dados demonstraram queda de consumo de $15 \%$ a $30 \%$ para o arroz e de 16\% a 38\% para o feijão entre os anos de 1974 a 1996. Alguns alimentos apresentaram grande variação positiva, como a ocorrida com o queijo, com aumento de consumo de $222 \%$, observado de maneira mais expressiva no Rio de Janeiro. Para o frango, há variações positivas entre 92\% e 163\% no mesmo período. Destaca-se a elevação acentuada no consumo de refrigerantes observada nos cinco municípios, sendo superior a 500\% em Campinas e em Goiânia, e a de salsicha em Campinas e Ouro Preto, no período de 1987 a 1996, com aumento de $324 \%$ e $550 \%$, respectivamente. Uma comparação quantitativa desse estudo com os dados obtidos no ENDEF (1974/1975) revela ampliação de consumo de alimentos, mas as dietas apresentam-se qualitativamente inadequadas (GALEAZZI et al., 1997).

Com os dados do Estudo Multicêntrico, verificou-se, ainda, que parcela importante da população se encontra fora das faixas de recomendação de ingestão de micronutrientes. Entre as mulheres cariocas, $44 \%$ e $66 \%$ apresentaram consumo deficiente de ferro e cálcio, respectivamente. A análise do consumo de ferro em Curitiba demonstrou inadequação para $33 \%$ das mulheres e $12 \%$ dos homens. Com relação ao cálcio, grande parte da população apresenta consumo aquém do recomendado, variando de 50\% a $80 \%$ em Ouro Preto e Goiânia. A ingestão de 
gordura manteve-se próxima ou inferior ao recomendado (30\% do valor energético total da dieta), mas observou-se consumo desequilibrado de gordura saturada e de colesterol. Mais de $40 \%$ de homens e mulheres consumiam quantidades superiores a 300mg de colesterol por dia nas cidades do Rio de Janeiro, de Curitiba e de Campinas (GALEAZZI et al., 1997).

O consumidor brasileiro, principalmente o dos grandes centros urbanos, vem seguindo as mesmas tendências alimentares de países industrializados, preferindo alimentos semiprontos a produtos que exigem tempo e trabalho no preparo (ABREU, 2000). A falta de tempo para o preparo das refeições em casa e a crescente preocupação com a saúde e a qualidade de vida têm sido grandes motivadores desse contexto (GARCIA, 1999; SILVA et al., 2002). Outros fatores que agilizaram a mudança foram: a entrada da mulher no mercado de trabalho, reestruturando os hábitos alimentares da família; a abertura do mercado comercial, que facilita importações; o aumento do poder aquisitivo da população de baixa renda, que expandiu o potencial de compra e consumo; as dificuldades do transporte e a necessidade de ganhar tempo (OLIVEIRA et al., 1996).

O mercado de alimentos semiprontos está em franca expansão e já representa um dos segmentos do setor de alimentação que mais crescem no mundo. A comida semipronta apresenta, como característica principal, a praticidade, que nem sempre é acompanhada por qualidade nutricional (NESTEL et al., 1996). Nos anos 90, a produção de alimentos congelados aumentou em 126\%, e a importação de produtos industrializados teve crescimento de 409\% (GARCIA, 1999), o que demonstra aumento expressivo no consumo de alimentos que agilizam o tempo de preparo.

Segundo dados da Associação Brasileira das Indústrias de Alimentação (ABIA), citados por Nantes e colaboradores (1999), a venda de alimentos semiprontos cresceu $16 \%$ em 1997, ao passo que a venda total de alimentos aumentou apenas 1,1\% no mesmo período. Na última Pesquisa de Orçamento Familiar (POF-1996), o gasto com "alimentos preparados" e "enlatados e conservas" somavam cerca de $3 \%$ da despesa média mensal familiar em alimentação (IBGE, sd) e acredita-se que a tendência seja de aumento desse valor.

Estudos confirmam a relação positiva entre refeições fora de casa e obesidade, especialmente tratando-se do tipo fast food (BINKLEY et al., 2000; MONTEIRO et al., 2000; GREENE et al., 2001). Calcula-se que os custos mundiais com o tratamento do excesso de peso sejam duas vezes maiores do que a receita total obtida pela indústria de fast food (SCHLOSSER, 2001).

Um dos fatores que agilizou o crescimento do food service, isto é, de refeições fora do lar e do consumo de alimentos de rápido preparo foi a entrada da mulher no mercado de trabalho, que em 1971 correspondia a apenas $23 \%$ da popu- 
lação economicamente ativa e, em 2000, a 51\%. Com isso, o tempo disponível para o preparo das refeições passou de 2 horas para 15 minutos, o que reestruturou os hábitos alimentares da família e a sua estrutura, já que em 1998, 25,9\% das mulheres eram chefes de família (ABIA, 2002).

Dados de 2002 da ABIA demonstram que, desde a implantação do Plano Real até 2001, houve crescimento de 190,7\% dos setores ligados ao food service e de $16,5 \%$ de alimentação fora do lar. O número de restaurantes comerciais no Brasil duplicou na última década e movimentou $\mathrm{R} \$ 5$,2 bilhões de reais em 2001; o mesmo valor foi obtido com a venda de alimentos em lanchonetes e fast foods (ABIA, 2002).

Nos Estados Unidos, metade das refeições são feitas fora de casa e, no Brasil, isso acontece com uma em cada quatro refeições nos grandes centros urbanos; mas a tendência de crescimento é praticamente a mesma (ABIA, 2002; NANTES et al., 1999). A POF realizada pelo IBGE em 1996 confirma essa situação ao demonstrar que os gastos com "alimentação fora do domicílio" correspondiam a 21,3\% das despesas totais com alimentação; nos grandes centros, esse valor ultrapassava $25 \%$, pois o item "almoço e jantar fora de casa" atingiu 9,3\% das despesas com alimentos (ABIA, 2002; IBGE, 2002).

Os órgãos públicos de fiscalização e os profissionais envolvidos na área de alimentos, alimentação e nutrição exercem importante papel no processo de conhecimento das tendências de consumo e devem atuar nos diferentes setores do sistema agroindustrial para garantir a qualidade dos produtos e, conseqüentemente, a qualidade de vida da população (MEGIDO \& XAVIER, 1995).

A escassez de informação sobre consumo de alimentos no País e a ausência de dados sobre hábitos alimentares tornam imprescindíveis novos estudos que verifiquem as tendências de consumo de alimentos, bem como o impacto desses comportamentos nos aspectos nutricionais e de saúde da população brasileira. 
Tabela 1 - Fatores que influenciam o comportamento alimentar.

Alimentos produzidos

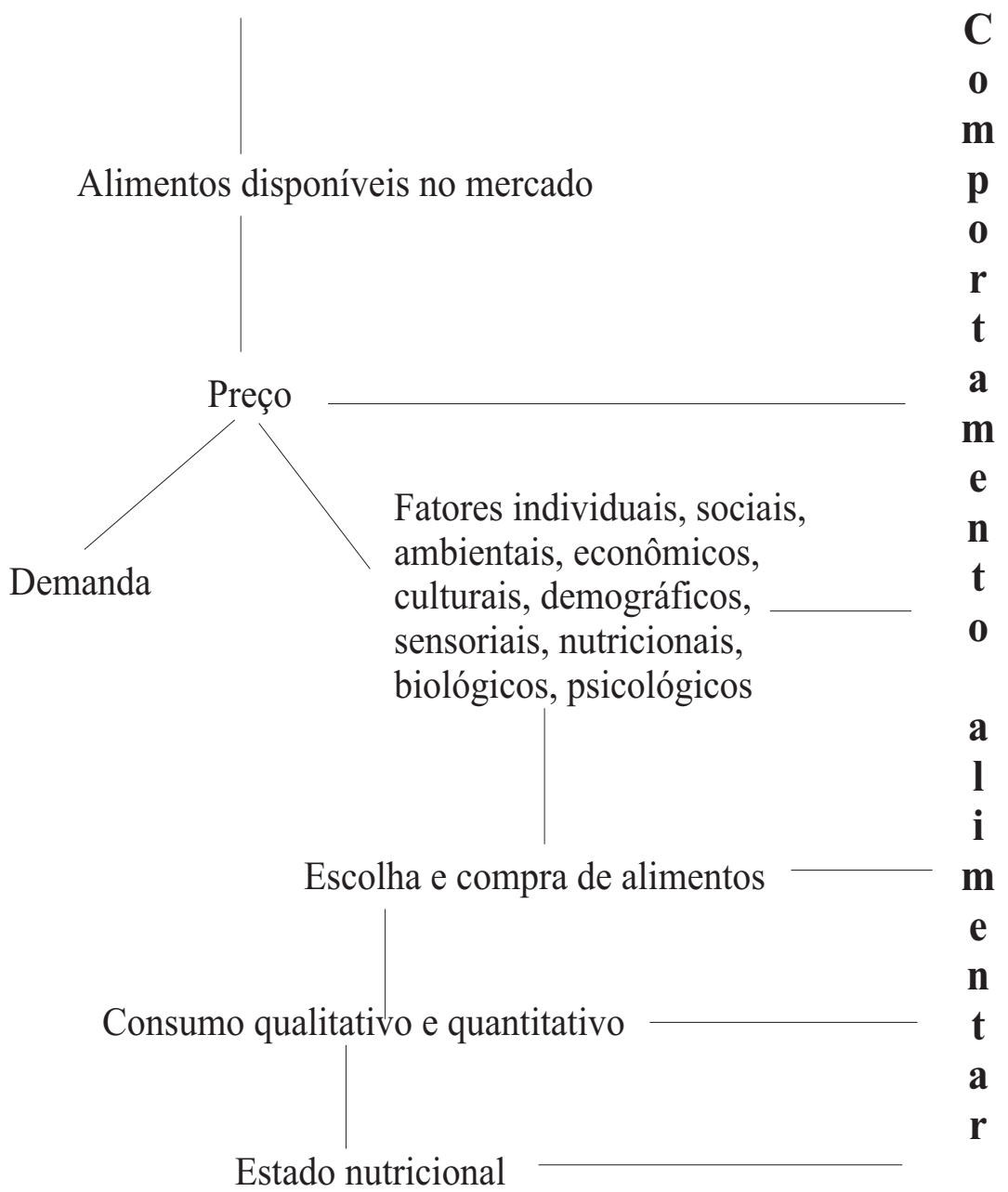

Adaptado de Castro (1995). 


\section{Referências}

[ABIA] Associação Brasileira de Indústrias de Alimentação. O mercado de food service no Brasil. São Paulo: Presiden/Brasil Food 2003, 2002.

ABREU, E.S. Restaurante "por quilo": vale quanto pesa? Uma avaliação do padrão alimentar em restaurantes de Cerqueira César, São Paulo, SP. Dissertação de Mestrado - Faculdade de Saúde Pública da USP. São Paulo, 2000.

[ADA] American Dietetic Association. "Nutrition education for the public: Position of ADA”.In: J Am Diet Assoc 1996; 96: 1183-7.

Anding JD, Suminski RR, Boss L. "Dietary intake, body mass index, exercise and alcohol: are college women following the dietary guidelines for Americans?" In: J Am Coll Health 2001; 49 (4): 167-71.

Barreto SAJ, Cyrillo DC. "Análise da composição dos gastos com alimentação no Município de São Paulo (Brasil) na década de 1990”. In: Rev Saúde Pública 2001; 35 (1): 52-7.

Binkley JK, Eales J, Jekanowski M. “The relation between dietary change and rising US obesity”. In: Int J Obes Relat Metab Disord 2000; 24 (8): 1032-9.

Boog MCF. "Educação nutricional: passado, presente, futuro". In: Rev Nutr PUCCAMP 1997; 10 (1): 5-19.

Casotti L, Ribeiro A, Santos C, Ribeiro P. "Consumo de alimentos e nutrição: dificuldades práticas e teóricas”. In: Cadernos de debate 1998; VI: 26-39.

Castro IRR. Vigilância alimentar e nutricional: limitações e interfaces com a rede de saúde. Rio de Janeiro: Fiocruz; 1995. p. 26-27.

[CFN] Conselho Federal de Nutrição. Nutricionistas e o Programa Fome Zero [documento on line] 2003. Disponível em <URL: www.cfn.org.br>. [2003 Mar 27].

Connors M, et al. "Managing values in personal food systems". In: Appetite 2001; 36 (3): 189-200.

Contento IR, Randell JS, Basch CE. "Review and analysis of evaluation measures used in Nutrition Education intervention research". In: JNEB 2002; 34: 2-25.

Cotugna N, Subar MF, Heimendinger J, Kahle L. "Nutrition and cancer prevention knowledge, beliefs, attitudes and practices": The 1987 National Health Interview Survey. In: $J$ Am Diet Assoc 1992; 92 (8): 963-8.

De Castro JM. “Socio-cultural determinants of meal size and frequency”. In: Br J Nutr 1997; 77, Suppl 1: S39-S55.

De Oliveira SP, Thébaud-Mony A. "Estudo do consumo alimentar: em busca de uma abordagem multidisciplinar”. In: Rev Saúde Pública 1997; 31 (2): 201-8.

Doyle EL, Feldman RH. "Factors affecting nutrition behavior among middle-class adolescents in urban area of Northern region of Brazil’. In: Rev Saúde Pública 1997; 31 (4): 34250 .

Drewnowski A, Popkin BM. "The nutrition transition: new trends in the global diet". In: Nutr Rev 1997; 55 (2): 31-43. 
[FAO/WHO] Food and Agriculture Organization/World Health Organization. International Conference on Nutrition. Major issues for nutrition strategies. Rome: FAO/WHO, 1992.

Freeland-Graves J, Nitzke S. "Position of the American Dietetic Association: total diet approach to communicating food and nutrition information". In: J Am Diet Assoc 2002; 102 (1): 100-8.

French SA, Story M, Jeffery RW. "Environmental influences on eating and physical activity". In: Annu Rev Public Health 2001; 22: 309-35.

Galeazzi MAM, Domene SMA, Sichieri R. "Estudo multicêntrico sobre consumo alimentar”. In: Cadernos de debate. NEPA/UNICAMP. Volume especial. 1997: 1-63.

Garcia, RWD. A comida, a dieta e o gosto: mudanças na cultura alimentar urbana. Tese de Doutorado em Psicologia - Instituto de Psicologia da USP. São Paulo, 1999.

Gibney MJ, Kearney M, Kearney JM. "IEFS pan-EU survey of consumer attitudes to food, nutrition and health". In: Eur J Clin Nutr 1997; 51 Suppl 2: 1-59.

Goldberg JP. "Nutrition communication in the $21^{\text {st }}$ Century: What are the challenges and how can we meet them?" In: Nutrition 2000; 16 (7/8): 644-6.

Greene HL, Prior T, Frier HI. "Foods, health claims and the law: comparisons of the United States and Europe”. In: Obes Res 2001; 9 Suppl 4: 276-83.

Grogan SC, Bell R, Conner M. "Eating sweet snacks: gender differences in attitudes and behavior”. In: Appetite 1997; 28 (1): 19-31.

Holm L. "Cultural and social acceptability of a healthy diet”. In: Eur J Clin Nutr 1993; 47: 592-599.

[IBGE] Instituto Brasileiro de Geografia e Estatística. Porcentagem de despesa média mensal familiar em alimentação [tabela do Excel] sd. Disponível em <URL: ftp:// ftp.ibge.gov.br/ Orcamento Familiar/ Pesquisa de Orcamentos Familiares 1995 1996/> [2002 Dez 1].

[IFIC] International Food Information Council. Center for Media and Public Affairs. Backgrouder: adult nutrition, health and physical activity. 1998. Available from <URL: http://ificinfo.health.org> [2002 Nov 22].

Jeffery RW, French SA. "Socioeconomic status and weight control practices among 20 to 45 year-old women”. In: Am J Public Health 1996; 86 (7): 1005-10.

Kearney JM, McElhone S. "Perceived barriers in trying to eat healthier: results of a pan-Eu consumer attitudinal survey". In: British Journal of Nutrition 1999; 81 Suppl 2: S133-S137.

Kelder SH et al. "Community-wide youth nutrition education: long-term outcomes of the Minnesota Heart Health Program”. In: Health Educ Res 1995; 10 (2): 119-31.

Koikkalainen M, Lappalainen R, Mykkanen H. "Why cardiac patients do not follow the nutritionist's advice: barriers in nutritional advice perceived in rehabilitation". In: Disabil Rehabil 1996; 18 (12): 619-23.

Kristal AR et al. "Psychosocial correlates of healthful diets: baseline results from the Working Well Study”. In: Prev Med 1995; 24 (3): 221-8. 
Lappalainen R et al. "Difficulties in trying to eat healthier: descriptive analysis of perceived barriers for healthy eating". In: Eur J Cl Nutr 1997; 51 Suppl 2: 36-40.

Lennernäs et al. "Influences on food choice perceived to be important by nationallyrepresentative samples of adults in the European Union". In: Eur J Clin Nutr 1997; 51 Suppl 2: 8-15.

Megido JLT, Xavier C. Marketing e agrobusiness. 2. ed. São Paulo: Atlas, 1995.

Mennell S, Murcott A, Van Otterloo AH. The Sociology of food: eating diet and culture. London: Sage Publications; 1992.

Milligan RA et al. "Heath-related behaviours and psycho-social characteristics of 18 yearold Australians". In: Soc Sci Med 1997; 45 (10): 1549-62.

[MS] Ministério da Saúde. Instituto Nacional de Alimentação e Nutrição. Fundação Instituto Brasileiro de Geografia e Estatística. Instituto de Pesquisa Econômica Aplicada. Pesquisa Nacional sobre Saúde e Nutrição: resultados preliminares. 2 ed. Brasília (DF); 1990.

[MS] Ministério da Saúde. Sobrepeso e obesidade [documento on line]. 2002a. Disponível em <http://portal.saude.gov.br/alimentação/sobrepeso.cfm>. [29 nov 2002].

[MS] Ministério da Saúde. Secretaria de Políticas Públicas de Saúde. Política Nacional de Promoção da Saúde: documento para discussão. Brasília (DF); 2002b.

Mondini L, Monteiro CA. Mudanças no padrão de alimentação. In: Monteiro CA (Org). Velhos e novos males da saúde no Brasil: a evolução do país e de suas doenças. São Paulo: Hucitec, 2000. p. 79-89.

Monteiro CA, Mondini L, de Souza AL, Popkin BM. "Da desnutrição para a obesidade: a transição nutricional no Brasil". In: Monteiro CA (Org). Velhos e novos males da saúde no Brasil: a evolução do país e de suas doenças. São Paulo: Hucitec, 2000. p. 247-55.

Morreale JS, Schwartz NE. "Helping Americans eat right: Developing practical and actionable public nutrition education messages based on the ADA Survey of American Dietary Habits". In: J Am Diet Assoc 1995; 95 (2): 305-8.

Nantes JFD, Kanesiro MAB, Oliveira JED. "Tendências de consumo de alimentos”. In: Nutrição em pauta 1999; (35): 36-8.

Nestel P, Noakes M, Clifton P. "Fats of the food industry: implications for cholesterollowering”. In: Lipids 1996; 31 Suppl 65-9.

Oliveira JED, Cunha SFC, Marchini JS. A desnutrição dos pobres e dos ricos: dados sobre a alimentação no Brasil. São Paulo: Sarvier; 1996.

Oliveira SP. "Changes in food consumption in Brazil". In: Arch Latinoam Nutr 1997; 47 Suppl 2: 22-24.

Ornellas LH. A alimentação através dos tempos. 2. ed. Florianópolis: UFSC 2000. Antropologia cultural, culinária e nutrição, hábitos alimentares. p 272-95.

Puska P et al. "Changes in premature deaths in Finland: successful long-term prevention of cardiovascular diseases". In: Bull World Health Organ 1998; 76 (4): 419-25. 
Puska P. "Nutrition and mortality: the Finnish experience". In: Acta Cardiol 2000; 55 (4): 213-20.

Randall E, Sanjur D. "Food preferences: their conceptualization and relationship to consumption”. In: Ecol Food Nutr 1981; 11: 151-161.

Rozin P. Towards a psychology offood choice. Bruxelles: Institut Danone; 1998. Human food selection: the interaction of biology, culture and individual experience. p. 59-90.

Rozin P, Schulkin J. "Food selection”. In: Rozin P. Towards a psychology offood choice. Bruxelles: Institut Danone; 1998. p. 17-58.

Schlosser E. País fast food: o lado nocivo da comida americana. São Paulo: Ática; 2001.p. 327.

Shepherd R. "Factors influencing food preferences and choice". In: Handbook of the psychophysiology of human eating. Wiley psychophysiology handbooks. Chichester: Gale; 1989. p 3-24.

Silva DO, Recine EGIG, Queiroz EFO. "Concepções de profissionais de saúde da atenção básica sobre a alimentação saudável no Distrito Federal, Brasil”. In: Cad Saúde Pública 2002; 18 (5): 1367-77.

Smith AM, Owen N. "Associations of social status and health-related beliefs with dietary fat and fiber densities". In: Prev Med 1992; 21 (6): 735-45.

Spear HI, Kulbok PA. "Adolescent health behaviors and related factors: a review”. In: Public Health Nurs 2001; 18 (2): 82-93.

Steptoe A, Pollard TM, Wardle J. "Development of a measure of the motives underlying the selection of food: the food choice questionnaire". In: Appetite 1995; 25 (3): 267-84.

Taddei JA. “Epidemiologia”. In: Nóbrega FJ. Distúrbios da nutrição. Rio de Janeiro: Revinter; 1998. p. 384-386.

Thompson RL et al. "The Health Education Authority's health and lifestyle survey 1993: who are the low fruit and vegetable consumers?" In: J Epidemiol Community Health 1999; 53 (5): 294-9.

[USDA] United States Department of Agriculture. Food Surveys Research Group. Beltsville Human Nutrition Research Center. Agricultural Research Service. Results from USDA' s 1994-96 Diet and Health Knowledge Survey. 1998.

Vaandrager HW, Koelen MA. "Consumer involvement in nutritional issues: the role of information”. In: Am J Clin Nutr 1997; 65 Suppl 6: 1980-1984.

[WHO] World Health Organization. Diet, nutrition and the prevention of chronic diseases. Report of a WHO study group. WHO Technical Report Series 797. Geneva; 1990.

[WHO] World Heatlh Organization. Health report 2002 [document on line] 2002. Available from <URL: $\underline{\text { www. who.int }}>$ [2002 Nov 22].

[WHO/FAO] World Health Organization/Food and Agriculture Organization. Diet, nutrition and the prevention of chronic diseases. Report of a joint WHO/FAO expert consultation. WHO Technical Report Series 916. Geneva; 2003.

Zunft HJF et al. "Perceived benefits of healthy eating among nationally-representative sample of adults in the European Union". In: Eur J Clin Nutr 1997; 51 Suppl 2: 41-6. 